\title{
An adaptive filtering method based on EMD for X-ray pulsar navigation with uncertain measurement noise
}

\author{
N. Li ${ }^{1}$, Z. W. Kang ${ }^{1}$, J. Liu ${ }^{2}$ and X. M. Xu ${ }^{1}$ \\ ${ }^{1}$ College of Information Science and Engineering, Hunan University, Changsha 410082, China \\ ${ }^{2}$ College of Information Science and Engineering, Wuhan University of Science and Technology, Wuhan \\ 430081, China
}

\begin{abstract}
Affected by the unstable pulse radiation and the pulsar directional errors, the statistical characteristics of the pulsar measurement noise may vary with time slowly and cannot be accurately determined, which cause the filtering accuracy of the extended Kalman filter(EKF) in pulsar navigation positioning system decline sharply or even diverge. To solve this problem, an adaptive extended Kalman filtering algorithm based on the empirical mode decomposition(EMD) is proposed. In this method, the high frequency noise is separated from measurement information of pulsar by the method of EMD, and the noise variance can be estimated to update the parameters of EKF. The simulation results demonstrate that compared with conventional EKF, the proposed method can adaptively track the change of the measurement noise, and still keeps high estimation accuracy with unknown measurement noise, the positioning accuracy of the pulsar navigation is improved simultaneously.
\end{abstract}

\section{Introduction}

$\mathrm{X}$-ray pulsar navigation is an emerging autonomous navigation technology for spacecraft, which can provide high precision navigation information[1-3]. X-ray pulsars are neutron stars that spin rapidly and emit stable pulsed radiation signal. Based on the cumulative processing of the pulsed radiation signal, the pulse time of arrival (TOA) is obtained and be utilized as the basic measurement of the pulsar-based navigation[4-6]. Combining the measurement information with orbital dynamics equation, the accurate positioning of the spacecraft can be achieved by the method of optimal estimation[7].

At present, the EKF is the most widely used filtering method in the study of autonomous navigation of spacecraft based on X-ray pulsars[8-9]. when a prior information is given and the model parameters are known, EKF can get better positioning accuracy[10]. However, affected by the unstable pulse radiation, the pulsar background noise and the pulsar directional errors, the statistical characteristics of the pulsar measurement noise may vary with time slowly and cannot be accurately determined limited to the current measurement equipment [11-12]. Scince EKF can not adjust to the change of the measurement noise, then the filtering accuracy of EKF will decline or even diverge. To this end, many scholars have proposed a variety of adaptive Kalman filtering algorithm which mainly concentrated in two aspects: adaptive filtering based on innovation and multiple model

Corresponding author: Z. W. Kang <hn_zwkang@126.com> 
estimation(MME)[13-17]. The former can adjust the covariance matrix of measurement noise by introducing the adjustment factor, and the calculation is simple but the filtering accuracy is limited. The MME uses multiple filters with different parameters simultaneously, although the accuracy has been improved, but the calculation is rather complex.

Based on the previous studies, combing the EMD with the EKF, an adaptive filtering method based on EMD_EKF is proposed in this paper. The pulsar measurement model with unknown parameters is established and the noise component can be extracted from the measurement information by meas of EMD. Then, the noise variance is estimated and the relevant parameter of the EKF algorithm is modified in real time to realize the state estimation of the spacecraft.

\section{Pulsar navigation model}

\subsection{The obit dynamic model}

This paper utilizes the transfer orbit to illustrate, assuming the position vector of the spacecraft $\boldsymbol{r}=[x, y, z]^{T}$ and the velocity vector $\boldsymbol{v}=\left[v_{x}, v_{y}, v_{z}\right]^{T}$, the orbit dynamic equation can be suggested as:

$$
\left[\begin{array}{c}
\dot{r} \\
v
\end{array}\right]=\left[\begin{array}{l}
v \\
a
\end{array}\right]+\left[\begin{array}{l}
w_{r} \\
w_{v}
\end{array}\right]
$$

Assuming system state variable $\mathbf{x}=\left[x, y, z, v_{x}, v_{y}, v_{z}\right]^{T}$, noise variable $\boldsymbol{w}(t)=\left[0,0,0, \Delta f_{x}, \Delta f_{y}, \Delta f_{z}\right]^{T}$ Then (1) can be written as:

$$
\dot{X}=\left[\begin{array}{l}
v \\
\alpha
\end{array}\right]=\mathrm{f}(X, \mathrm{t})+w(\mathrm{t})
$$

$\boldsymbol{\alpha}$ is acceleration vector of spacecraft, since the spacecraft is mainly affected by the gravity of the Sun, Mars, Earth and Jupiter in the transfer orbit, the specific forms of $\alpha$ can be written as:

$$
\dot{v}=\boldsymbol{a}=-u_{s} \frac{\boldsymbol{r}}{r^{3}{ }_{p s}}-u_{m}\left[\frac{\boldsymbol{r}-\boldsymbol{r}_{\boldsymbol{1}}}{r^{3}{ }_{p m}}+\frac{\boldsymbol{r}_{\boldsymbol{1}}}{r^{3}{ }_{s m}}\right]-u_{e}\left[\frac{\boldsymbol{r}-\boldsymbol{r}_{\mathbf{2}}}{r^{3}{ }_{p e}}+\frac{\boldsymbol{r}_{\mathbf{2}}}{r^{3}{ }_{s e}}\right]-u_{j}\left[\frac{\boldsymbol{r}-\boldsymbol{r}_{\mathbf{3}}}{r^{3}{ }_{p j}}+\frac{\boldsymbol{r}_{\mathbf{3}}}{r^{3}{ }_{s j}}\right]+\boldsymbol{\Delta} \boldsymbol{f}
$$

Specific parameters refer to the reference[18].

\subsection{The measurement model of X-ray pulsar with uncertain noise}

The basic measurement of X-ray pulse navigation is the pulse TOA. The measurement model based on the pulse TOA deviation can be written as:

$$
\Delta t=t_{s s b}-t_{b}=\frac{1}{c} \boldsymbol{n} \cdot \boldsymbol{r}
$$

Where, $t_{s s b}$ and $t_{b}$ respect the pulse TOA at the spacecraft and SSB . $\boldsymbol{n}$ and $\boldsymbol{r}$ are the pulsar direction vector and the spacecraft position vector respectively, and $c$ represents the light speed. Assuming the pulsar observation vector as $z=[c \cdot \Delta t]$, then the pulsar navigation measurement equation can be presented as follows: 


$$
z=h(\boldsymbol{X}(t), t)+v
$$

Here, $v$ is the pulsar measurement noise with the standard deviation of $\sigma$ which can be calculated according to Taylor formula[19] .

$$
h[X(t), t]=\boldsymbol{n} \cdot \boldsymbol{r}
$$

The navigation accuracy of the pulsar navigation system is depends on the accuracy of the TOA, but due to exists of the unstable pulse radiation, the background noise and the pulsar directional errors the pulsar measurement will be inevitably disturbed. The interference mentioned above can be classified as the pulsar measurement noise which is regrade as white Gaussian noise, and the noise variance can not be calculated limited to the current measurement equipment and technology. The measurement equation is:

$$
\begin{aligned}
& z=h(X(t), t)+v^{\prime} \\
& v^{\prime}=v+\xi
\end{aligned}
$$

Assuming that the variance of $v^{\prime}$ is $\sigma^{\prime}$, then $\sigma^{\prime}$ is the unknown parameter of pulsar measurement model. This paper employs three pulsars as navigation pulsar and the observation vector and measurement model of pulsar can be recorded as:

$$
\begin{gathered}
\boldsymbol{Z}=\left[z_{1}, z_{2}, z_{3}\right]^{T} \\
\boldsymbol{Z}(t)=h(\boldsymbol{X}, t)+\boldsymbol{V}^{\prime}
\end{gathered}
$$

where

$$
\begin{gathered}
h(\boldsymbol{X}, t)=\left[h_{1}[\boldsymbol{X}(t), t], h_{2}[\boldsymbol{X}(t), t], h_{3}[\boldsymbol{X}(t), t]\right]^{T} \\
\boldsymbol{V}^{\prime}=\left[v_{1}{ }^{\prime}, v_{2}{ }^{\prime}, v_{3}{ }^{\prime}\right]^{T}
\end{gathered}
$$

Here, $\boldsymbol{V}^{\prime}$ is the system measurement noise and the corresponding covariance matrix $\boldsymbol{R}^{\prime}$ which is the system parameter to be estimated can be expressed as :

$$
\boldsymbol{R}^{\prime}=\left[\sigma_{1}^{\prime}, \sigma_{2}^{\prime}, \sigma_{3}^{\prime}\right]^{T}
$$

\section{Adaptive filtering algorithm based on empirical mode decomposition}

As the models of the navigation system are nonlinear, the EKF is adopted as the navigation filter to realize continuous orbit determination of the spacecraft[8,20]. However, when the statistical characteristics of the measurement noise varies, the traditional EKF can not adjust to the change, which may cause serious estimation bias. This paper introduce the EMD to the the EKF, the highfrequency noise component can be extracted from the pulsar measurement. Then the noise variance is estimated to adjust the relevant parameters in EKF constantly to achieve the optimal state estimation of the spacecraft.

\subsection{The empirical mode decomposition}


The NAE member N.E. Huang has proposed the empirical mode decomposition method(EMD), which is a new signal processing method that apply for nonlinear processes[21]. According to its own characteristics, the signal is decomposed into several intrinsic mode functions (IMF) which represent different frequency components of the signal. In addition, $\mathrm{Wu}$ and Huang has found that each IMF component obtained by decomposing white noise via empirical mode decomposition satisfy the normal distribution. Therefore, the EMD method can be used to decompose the noisy signal with white noise.

After decomposed by EMD, the noisy signal can be resolved into several IMF components, which can be expressed as[22]:

$$
x(t)=\sum_{i=1}^{n} c_{i}(t)
$$

Where $x(t)$ represent the noisy signal, $c_{i}(t)$ is the IMF components which represent different frequency components of the signal and $n$ is the number of the IMF. According to the correlation of white noise, we can determine whether the component is the desired noise component by calculating the auto-correlation or cross-correlation of these IMF components, and then noise variance can be calculated.

\subsection{The filtering algorithm of EMD_EKF}

Discretizing (2) and (10), the discrete system model is obtained as follows:

$$
\begin{aligned}
& \boldsymbol{X}(k+1)=\boldsymbol{F}(k+1, k) \boldsymbol{X}(k)+\boldsymbol{W}(k) \\
& \boldsymbol{Z}(k+1)=\boldsymbol{H}(k+1) \boldsymbol{X}(k+1)+\boldsymbol{V}^{\prime}(k+1)
\end{aligned}
$$

Where $\boldsymbol{X}$ is the state vector of the system and $\boldsymbol{Z}$ is the measurement vector of the pulsar, $\boldsymbol{F}$ is the system state transition matrix and $\boldsymbol{H}$ is the observation matrix. $\boldsymbol{W}$ is the state noise matrix with variance of $\boldsymbol{Q}$ and $\boldsymbol{V}^{\prime}$ is the observation noise matrix with unknown variance $\boldsymbol{R}^{\prime}$. Because the measurement noise variance is unknown, so the observed signal should be deposed by EMD before using the EKF to update the state of the spacecraft. The specific filtering process is as follows:

1) Calculating the one step prediction value $\hat{\boldsymbol{X}}_{k+1, k}$ of the state and error variance matrix $P_{k+1 . k}$

2) Calculating one step prediction of measurement: $\hat{\boldsymbol{Z}}_{k+1, k}=\boldsymbol{H}_{k+1} \hat{\boldsymbol{X}}_{k+1, k}$

3) Calculating one step prediction of covariance matrix: $\boldsymbol{P}_{k+1, k}=\boldsymbol{F}_{k+1, k} \boldsymbol{P}_{k, k} \boldsymbol{F}_{k+1, k}{ }^{T}+\boldsymbol{Q}_{k+1}$

4) Deposing the measurement by the method of EMD, assuming that the first $\mathrm{K}(\mathrm{K}<\mathrm{N})$ term is the noise component, the noise variance is calculated as: $\boldsymbol{R}^{\prime}{ }_{k+1}=\sum_{i=1}^{K} I M F_{i}$

5) Calculating the gain matrix: $\boldsymbol{K}_{k+1}=\boldsymbol{P}_{k+1, k} \boldsymbol{H}_{k+1}\left[\boldsymbol{H}_{k+1} \boldsymbol{P}_{k+1, k} \boldsymbol{H}_{k+1}^{T}+\boldsymbol{R}_{k+1}^{\prime}\right]^{-1}$

6) Updating state estimation: $\hat{\boldsymbol{X}}_{k+1}=\hat{\boldsymbol{X}}_{k+1, k}+\boldsymbol{K}_{k+1}\left(\boldsymbol{Z}_{k+1}-\hat{\boldsymbol{Z}}_{k+1, k}\right)$

7) Updating covariance matrix: $\boldsymbol{P}_{k+1}=\left[\boldsymbol{I}-\boldsymbol{K}_{k+1} \boldsymbol{H}_{k+1}\right] \cdot \boldsymbol{P}_{k+1, k}$

\section{Simulation results and analysis}

The Mars explorer orbit data of the "Pathfinder Mars" is adopted in this paper, the specific simulation conditions refer to the reference[5].There are two kinds of measurement noise used in simulation: 
white Gaussian noise with fixed variance $\sigma$ and time-varying noise whose variance intensity changed on the basis of the fixed variance. In the above two kinds of noise, the EKF and EMD_EKF method are used to estimate the state of the spacecraft, and the navigation performance is analyzed.

Figure 1 shows the simulation results of EKF based on those two noises described above. As can be seen from Figure 1, EKF can achieve a good filtering effect with fixed noise. But when the variance of measurement noise vary with time, the EKF cannot track and adjust the changes, which result in a sharp decline in the navigation accuracy or even lead to filter divergence.

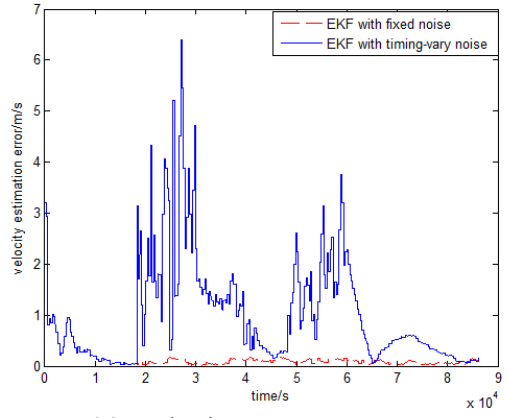

(a) velocity error

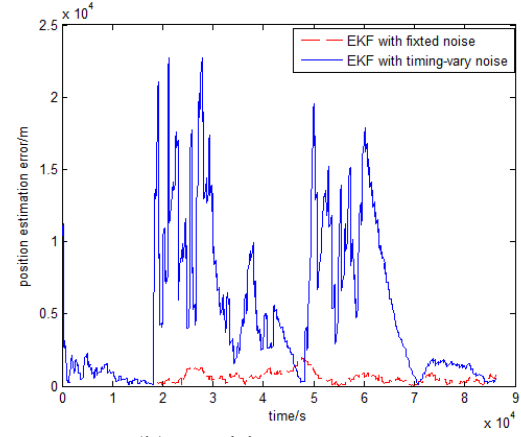

(b) position error

Figure 1. Simulation results of conventional EKF under two kinds of noise

Figure 2 shows the simulation results based on EKF and EMD_EKF with timing-vary noise. From Figure 2, we can see that when the variance of measurement noise varies, the positioning error of navigation based on EKF increases sharply because the variance of measurement noise adapted in EKF is inconsistent with the true value. The proposed adaptive filtering based on EMD_EKF adjust the noise variance adaptively and still maintain high accuracy of positioning when the noise variance is unknown. A further explanation of Figure 2 is given by table 1 , We can see that the positioning error of EKF algorithm has achieved to $3000 \mathrm{~m}$ and that error of this method is only $500 \mathrm{~m}$, the positioning accuracy is 5 times higher than that of EKF.

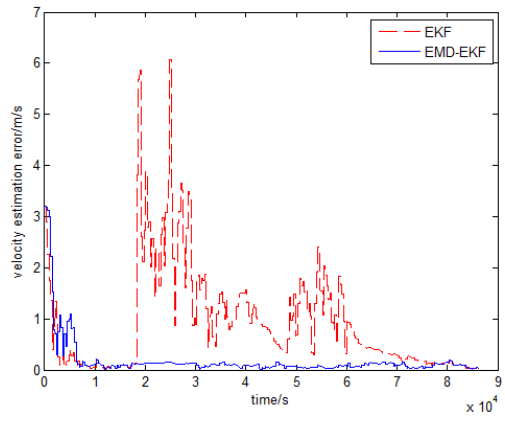

(a) velocity error

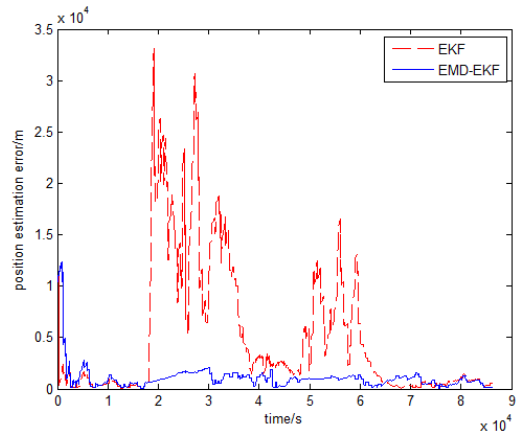

(b) position error

Figure 2. Filtering results of two methods with timing-vary noise 
Table 1. Performance comparison of two methods with unknown noise variance

\begin{tabular}{lll}
\hline Filter method & position error & velocity error \\
\hline EKF & $3538 m$ & $0.625 \mathrm{~m} / \mathrm{s}$ \\
EMD_EKF & $526 \mathrm{~m}$ & $0.0726 \mathrm{~m} / \mathrm{s}$ \\
\hline
\end{tabular}

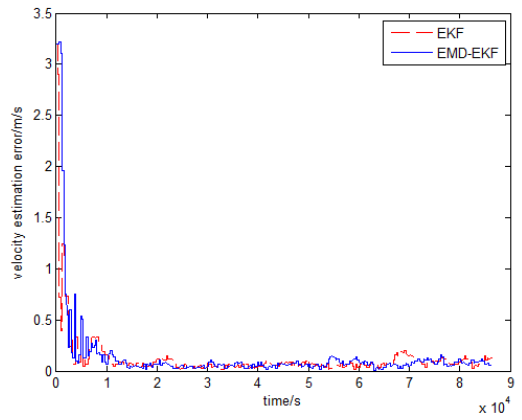

(a) velocity error
Table 2. Performance comparison of two methods with fixed noise variance

\begin{tabular}{lcc}
\hline Filter method & position error & velocity error \\
\hline EKF & $464 m$ & $0.0701 \mathrm{~m} / \mathrm{s}$ \\
EMD_EKF & $467 \mathrm{~m}$ & $0.0714 \mathrm{~m} / \mathrm{s}$ \\
\hline
\end{tabular}

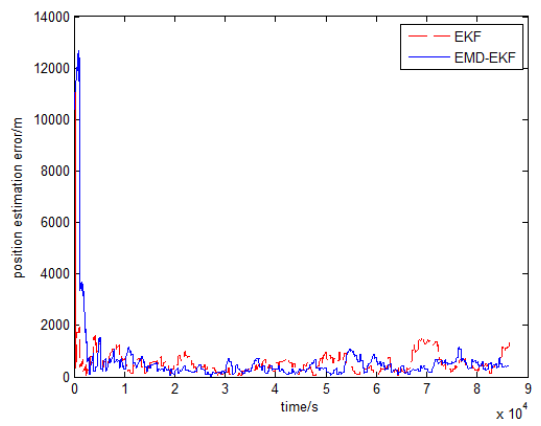

(b) position error

Figure 3. Filtering results of two methods with fixed noise

It can be seen from Figure 3 that the navigation accuracy of the two algorithms with fixed noise is consistent basically. It shows that the proposed method can obtain high positioning accuracy both in the fixed noise and unknown noise which can restrain the influence of unknown disturbance on navigation accuracy. Table 2 shows the performance comparison of these two methods.

Figure 4 shows the comparison between the reconstructed noise after EMD decomposition and the original noise with fixed variance of pulsar B0531+21. We can see that the high-frequency part extracted from the pulsar measurement by means of EMD can reconstruct the original noise primely. Figure 5 suggests that the proposed method can adaptively track the change when the noise variance varies with time and ensure that the noise variance used in the EKF is consistent with the real value through estimating the noise variance in real time.
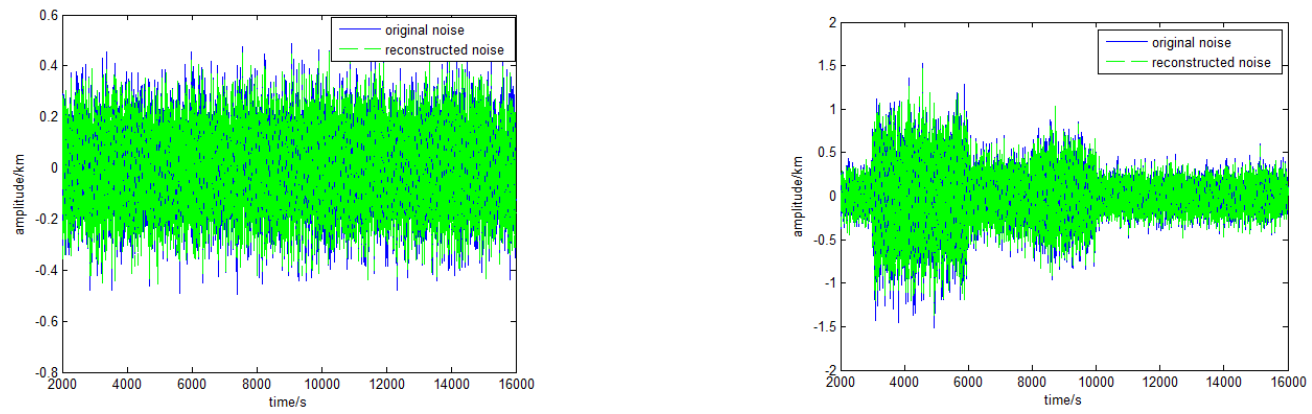

Figure 4. Comparison of two noise with fixed variance

Figure 5. Comparison of two noise with timing-vary variance 


\section{Conclusion}

In this paper, an adaptive extended Kalman filtering method based on empirical mode decomposition is studied to solve the filtering problem of the X-ray pulsar-based navigation system with uncertain measurement noise. This method does not need to determine the statistical properties of the measurement noise in advance and can track the varies of the noise adaptively to modify the relevant parameters of EKF in real time. Compared with the conventional EKF, the proposed method can still maintain a high estimation accuracy with timing-vary and uncertain measurement noise. Thus it is an adaptive Kalman filtering algorithm that is more suitable for the spacecraft navigation in deep space environment in which the measurement noise is difficult to determine.

\section{References}

1. S.I. Sheikh, D.J. Pines, P.S. Ray, The use of X-ray pulsars for spacecraft navigation, Adv. Astronaut. Sci, 119 (2005) 105-119.

2. S.I. Sheikh, D.J. Pines, Recursive Estimation of Spacecraft Position and Velocity Using X-ray Pulsar Time of Arrival Measurements, J. Inst. Navig, 53 (2006) 149 - 166.

3. K. Xiong, C.L. Wei, L.D. Liu, Research on the autonomous navigation of satellite constellation using pulsars, J. Astronaut, 29 (2008) 545-566.

4. B.J. Fei, G.Z. Yao, J. Du, Some problem of research on X-ray pulsar-based navigation., J. Aca. Armor. Fro. Eng, 27 (2013) 1-8.

5. J. Liu, J.C. Fang, Z.H. Yang, X-ray pulsar/Doppler difference integrated navigation for deep space exploration with unstable solar spectrum, Aerosp. Sci. Technol., 41 (2015) 144 150.

6. P.Y. Cui, S. Wang, A. Gao, et al, X-ray pulsars/Doppler integrated navigation for Mars final approach, Adv. Space. Res. , 57 (2016) 1889-1900.

7. Y.D. Wang, W. Zheng, S.M. Sun, L. Li, X-ray pulsar-based navigation system with the errors in the planetary ephemerides for Earth-orbiting satellite, Adv. Space Res. 51 (2013) 2394-2404.

8. M.Y. Fu, Z.H. Deng, L.P. Yan, Kalman filtering theory and application in navigation system, Science Publishing House, 2010.

9. X.L. Ning, X. Ma, C. Peng, J.C. Fang, Analysis of filtering methods for satellite autonomous orbit determination using celestial and geomagnetic measurement, Math. Probl. Eng., 2 (2012) $75-78$.

10. J. Liu, Z.W. Kang, P. White, J. Ma, J.W. Tian, Doppler/XNAV integrated navigation system using small-area X-ray sensor, IET Radar Sonar Navig, 5 (2011) 1010 1017.

11. J. Liu, J. Ma, J.W. Tian, Z.W. Kang, P. White, X-ray pulsar navigation method for spacecraft with pulsar direction error, Adv. Space. Res., 46 (2010) 1409 1417.

12. X.L. Ning, Z. Li, P.P. Huang, Y.Q. Yang, An adaptive Kalman filter for Mars spacecraft acquisition phase, J. Deep. Space. Explor., 3 (2016)237-245.

13. M.J. Yu, INS/GPS integration system using adaptive filter for estimating measurement noise variance, IEEE Trans. Aerosp. Electron. Syst., 48 ( 2012) 1786-1792.

14. B.N. Alsuwaidan, J.L. Crassidis, Y. Cheng, Generalized multiple-model adaptive estimation using an auto correlation approach, IEEE Trans. Aerosp. Electron. Syst., 47 (2010) 2138-2152.

15. K. Xiong, T. Liang, Y.J. Lei, Multiple model Kalman filter for attitude determination of precision pointing spacecraft, Acta Astronau., 68 (2011) 843-852 .

16. K. Xiong, C.L. Wei, L.D. Liu, Robust multiple model adaptive estimation for spacecraft autonomous navigation, Aerosp. Sci. Technol., 42 (2015) 249 258.

17. F. Yang, Z. Wang, Y.S. Hung, Robust Kalman filtering for discrete time-varying uncertain systems with multiplicative noises, IEEE Trans. Autom. Control, 47 (2002) 1179-1183.

18. J. Liu, J.C. Fang, Z.W. Kang, J. Wu, X.L. Ning, Novel Algorithm for X-Ray Pulsar Navigation Against Doppler Effects, IEEE Trans. Aerosp. Electron. Syst., 51 (2015) 228 241.

19. J.H. Taylor, Pulsar timing and relativistic gravity, Philo-sophical Trans, R Soc. London, 341 (1992) 117-134. 
20. K. Xiong, C.L. Wei, L.D. Liu, Robust Kalman filtering for discrete-time nonlinear systems with parameter uncertainties, Aerosp. Sci. Technol. 18 (2012) 15-24.

21. N.E. Huang, S. Zheng, R. Steven, The empirical mode decomposition and the Hilbert spectrum for nonlinear non-stationary time Series Analysis, P. Math. Phys. Eng. Sci, london, 454 (1998) 903-995.

22. X.Y. Li, J. Jin, Y. Shen, Y.P. Liu, Noise level estimation method with application to EMD_based signal decomposing, J. Syst. Eng. Electron., 27 (2016) 763-771. 